\title{
Patient-specific finite element analysis of frictional behavior in different esophageal regions during endoscopy
}

\author{
ChengXiong LiN ${ }^{1}$, PAN REN $^{1}$, Wei Li ${ }^{1} *$, Hengyi DenG ${ }^{2}$, ZHONGRONG ZHOU ${ }^{1}$ \\ ${ }^{1}$ Tribology Research Institute, Key Laboratory for Advanced Technology of Materials of Ministry of Education, \\ Southwest Jiaotong University, Chengdu, China. \\ ${ }^{2}$ Department of General Surgery, Chengdu Second People's Hospital, Chengdu, China.
}

\begin{abstract}
Purpose: Endoscopy is a common and effective method to treat digestive system diseases. Not only can it detect the physiological state of the digestive tract, but also can conduct clinical operations. As a result, it's of great significance to make clear the relationship between the clinical operation and the complications. Methods: Considering the difficulty in measuring the contact force and determining the stress distribution in real time during endoscopy, a specific-patient finite element model for the frictional behavior at the endoscope-esophagus interface was built in current study. By collecting the CT data of the patient, a 3D esophagus model was built and divided into three characteristic regions (narrow region, thoracic region and abdominal region) according to the physiological structure. Results: Results showed that the radius of the narrowest position was the dominant factor for the maximum von Mises stress when the endoscope passed through the narrow region. For abdominal region and thoracic region, with the increasing coefficient of friction (COF) and amplitude, the total force duo to frictional force (CFSM), frictional dissipation (FD), strain energy (SE) and maximum von Mises stress (Max) all increased correspondingly. Meanwhile, the region of stress concentration gradually approached the initial contact stage. Conclusions: The results can provide theoretical basis and technical support for clinical application and offer some suggestions for medical workers during endoscopy as well.
\end{abstract}

Key words: human esophagus, frictional behavior, finite element model, patient-specific

\section{Introduction}

Endoscopy is a common and effective method to treat digestive system diseases. It enables the doctors not only to detect the physiological state of the digestive tract, but also conduct clinical operations, such as esophageal stent apposition [8], [10], extraction of esophageal foreign bodies [2], [17], biopsy sample [11] and so on. On the one hand, it plays a huge role in gastroesophageal diseases, but, on the other hand, it also brings some complications during or after endoscopy, which are easily ignored by people, especially the elderly. The complications mainly contain hyperemia, hemorrhage and perforations [1], [16].
At a result, it's of great significance to make the relationship between the clinical operation and the complications clear. In view of the difficulty in determining the contact force and stress distribution at the tissue-instrument interface, a practical finite element model is introduced [3], [22]. By simulating and simplifying the model to some extent, it can help medical workers improve the operation quality and offer suggestions for endoscopy.

Many researchers have carried out simulations studies on the soft tissue-medical instrument contact problem by using finite element model. These instruments mainly focus on intravascular stents [5], [9], [20], [25], [26], esophageal stents [12], [23], [30], aortic valves [4], [21], gastric band [7], etc. For example,

\footnotetext{
* Corresponding author: Wei Li, Tribology Research Institute, Key Laboratory for Advanced Technology of Materials of Ministry of Education, Southwest Jiaotong University, Chengdu 610031, China. Phone: +86-28-87634270, e-mail: liweijiani@home.swjtu.edu.cn

Received: January 4th, 2020

Accepted for publication: March 2nd, 2020
} 
Schiavone et al. [26] simulated the development of the latest commercial stent (Xience stent) inside a stenotic artery through three behaviors (expansion, dogboning and recoiling). Kajzer et al. [12] conducted the biomechanical analysis of the given geometrical form of esophageal stent used in the digestive tract and studied the stress and strain distribution of the specific elements in the modeled system. Carniel et al. [7] developed a computational tool to investigate the mechanical functionality of gastric conformations preoperatively and postoperatively. These articles mainly focused on the in situ evaluation of structural parameters and material properties of instruments (during and after deployment) as well as the stress distribution of tissue, but paid little attention to the frictional behavior at the soft tissue-instrument interface.

Meanwhile, with the rapid development of medical imaging techniques and computational methods, the patient-specific finite element model provides researchers with more realistic idea to study the target tissueinstrument behaviors. Morganti et al. [4] realistically reproduced the transcatheter aortic valve using a patient-specific finite element analysis and evaluated the impact of aortic root anatomy from two real patient datasets. He also conducted the isometric analysis on the model construction and simulation of aortic valve closure [21]. Auricchio et al. [3] studied the impact of carotid stent apposition on carotid artery anatomy by means of patient-specific finite element analysis. Magne et al. [19] introduced a rapid method for the generation of finite element models of dental structures and restoration with a Micro-CT scanner. Our research group also conducted experiments concerning the endoscope-esophagus interaction under different normal loads, sliding velocities, physiological structures and extension directions and made a series of conclusions [15], [16]. However, the friction experiment on esophageal tissue were performed in vitro.

Consequently, in order to better understand the interaction at the endoscope-esophagus interface, a patient-specific model based on the finite element model was used to study the frictional behavior in this paper. According to the physiological structure, the target esophagus was divided into three characteristic regions: narrow region, abdominal region and thoracic region. The study was organized as follows: first, we illustrated the generation of the patient-specific model of esophagus, and then introduced the basic parameter settings of the Abaqus model. Finally, we postprocessed the simulation outputs, and discussed the results.

\section{Materials and method}

\subsection{Model generation}

A 3-step procedure was followed to generate the 3D finite element model of the extracted human esophagus: Step 1: built the part of esophagus in Mimics based on the data of Micro-CT; Step 2: smoothed the surface of esophagus and measured the size of esophagus; Step 3: built the three-dimensional models of the esophagus
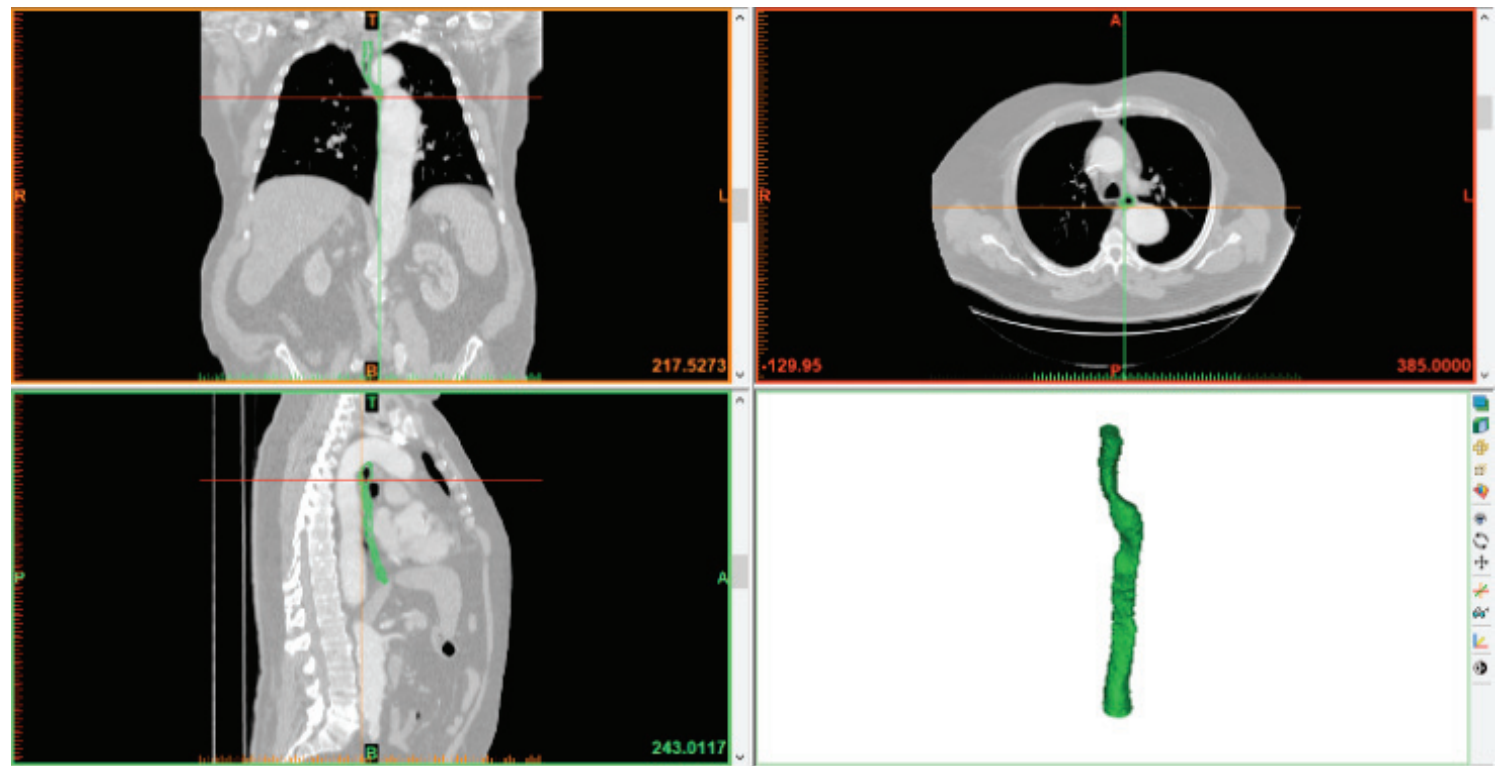

Fig. 1. The specific-patient esophagus opened in the Mimics in different perspectives 
in SolidWorks based on the previously measured esophageal size and exported them to Abaqus using a plugin (SIMULIA ABAQUS Interface for SolidWorks).

The esophagus data from Skyscan 1072 high resolution Micro-CT (Skyscan, Aartselaar, Belgium) was obtained from a 67-year-old male patient in the Department of General Surgery, Chengdu Second People's Hospital, Chengdu, China. The data was approved by the Ethics Committee of Sichuan Academy of Medical Sciences \& Sichuan Provincial People's Hospital, China. The scanning data was characterized by the following features: slice thickness was $3.75 \mathrm{~mm}$; slice increment was $2.5 \mathrm{~mm}$; slice width and height were $512 \times 512$ pixels; pixel spacing was $0.91 \mathrm{~mm}$; number of slices was 201 .

First, the origin data of esophagus from Micro-CT was opened using Mimics 20.0 (Materialise NV, Belgian). The first mask was built by adjusting the threshold of the software. By editing the previous mask layer-by-layer, the second mask was constructed to obtain the esophageal model accurately. Based on the second mask, the part of esophagus can be built through the calculation part in the software, as shown in Fig. 1.

Then, the portion of esophagus was smoothed to remove the burrs on the surface. The size of the esophagus can be measured in 3-Matic 12.0 which was a matching software with Mimics 20.0, as shown in Fig. 2. According to the physiological structure, three characteristic regions were chosen from top to bottom in the esophagus, namely narrow region, thoracic region and abdominal region. The basic size and shape were labelled in the Appendix. In addition, based on the size of these characteristic regions, the parts of esophagus can be built in Solidworks 2016 (Dassault system, USA).

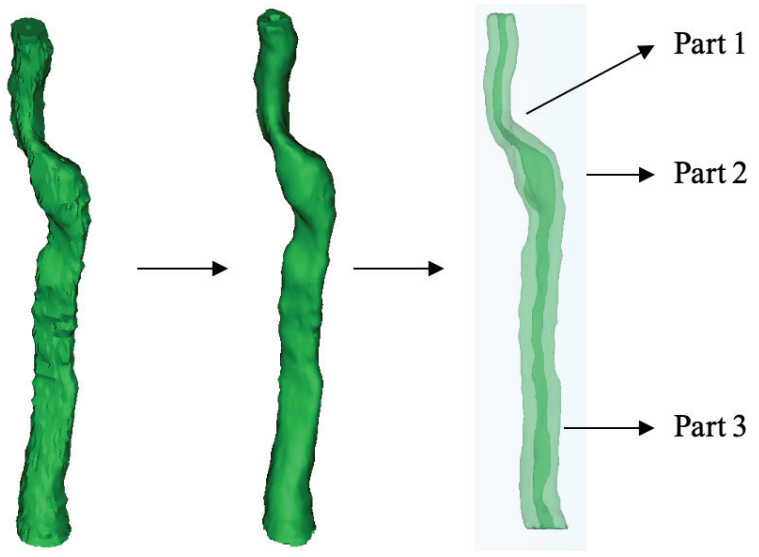

Fig. 2. The smoothing treatment and the subsequent measurement of the esophagus model
Finally, the esophagus parts were exported into Abaqus 2016 (Dassault Systemes, USA) via a plugin (SIMULIA ABAQUS Interface for SolidWorks), as shown in Fig. 3.

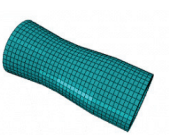

(a) Part 1

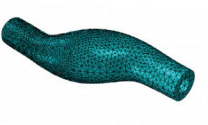

(b) Part 2

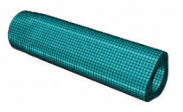

(c) Part 3

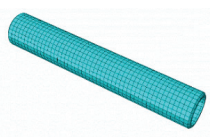

(d) Endoscope
Fig. 3. Three characteristic parts chosen from the esophagus model: part 1 - narrow region, part 2 - thoracic region, part 3 - abdominal region; endoscope

Assisted by the external force, the endoscope passed through the esophagus along the coaxial direction. The endoscope was simplified into a hollow cylindrical structure, as shown in Fig. 3d. In the initial stage, there was a buffer regions playing a guiding role when the endoscope contacted the esophagus [13]. The basic sizes of endoscope were also listed in Appendix. The part of endoscope was assembled with the esophagus in Abaqus to simulate the contact during endoscopy, as shown in Fig. 4. (a)

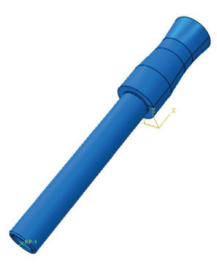

(c)

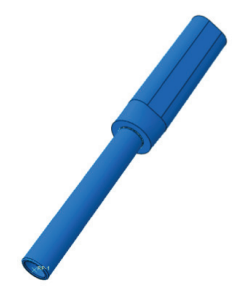

Fig. 4. The assembly of the endoscope and esophagus in Abaqus software

\subsection{Materials models and finite element procedure}

The number of elements, nodes as well as the type of element are listed in Table 1. Owing to the asymmetry of the thoracic region, the elements was set as C3D10M in order to better run the contact model in Abaqus. Meanwhile, the element density of the distal thoracic region was smaller than that of the proximal region to accelerate the calculation speed and save the calculation time.

Considering the viscoelasticity and incompressibility of the esophagus, the materials model was assigned with a linear elastic material, which was similar to a blood vessel [24]. The elastic modulus was $E=1.75 \mathrm{MPa}$, the Poisson's ratio was $\vartheta=0.49$ and the density of the esophagus was $\rho=1 \times 10^{3} \mathrm{~kg} / \mathrm{m}^{3}$ 
[23]. The endoscope was also a linear elastic material and the elastic modulus was $E=8.3 \mathrm{E} 5 \mathrm{MPa}$, the Poisson's ratio was $\vartheta=0.33$ and the density of the esophagus was $\rho=6.45 \times 10^{3} \mathrm{~kg} / \mathrm{m}^{3}$.

The model was opened as a static and general type when the endoscope started moving. The interaction between the endoscope and the esophagus was modelled by using surface-to-surface formulation. The coefficient of friction was 0.2 , which simulated the real frictional contact [19]. For boundary conditions, the endoscope was moving coaxially along the axial of esophagus to imitate the stable operation during endoscopy. Besides, the proximal end of the esophagus was constrained three displacement directions to avoid movements.

Table 1. the number of elements, the number of nodes as well as the type of the element

\begin{tabular}{|l|c|c|c|}
\hline $\begin{array}{c}\text { Name } \\
\text { of Part }\end{array}$ & $\begin{array}{c}\text { Number } \\
\text { of Elements }\end{array}$ & $\begin{array}{c}\text { Number } \\
\text { of nodes }\end{array}$ & $\begin{array}{c}\text { Type } \\
\text { of element }\end{array}$ \\
\hline Narrow region & 3780 & 5184 & C3D8R \\
\hline Thoracic region & 123696 & 180581 & C3D10M \\
\hline Abdominal region & 12000 & 14701 & C3D8R \\
\hline Endoscope & 1683 & 2964 & C3D8R \\
\hline
\end{tabular}

\subsection{Movement and analysis under different conditions}

The system locomotion was driven by setting the displacement of endoscope under the boundary condition and the displacement was $30 \mathrm{~mm}$ along the central axis of the esophagus. In order to overall understand the endoscope-esophagus interaction in the patient-specific model, the model consisted of three variable parameters: 1 . amplitude: the displacement of endoscope over a period of time; 2. coefficient of friction (COF): the coefficient of friction at the endoscope-esophagus interface; 3 . structure size: the diameter or length for esophagus. For the frictional behavior when the endoscope passed through the narrow region, the basic parameters of model are as follows: the radius and the length of the narrowest position are $3.5 \mathrm{~mm}$ and $5 \mathrm{~mm}$, respectively; the interfacial COF is 0.2 ; the amplitude of the model is $(0,0)-(1,1)$. When studying the influence of structural parameters, COF and amplitude factors on the frictional performance, only the corresponding parameter changes, while the other three parameters remain the same.

Besides, there were four characteristic indices to analyze the results of the model. For all simulations, only the job file of model was completed with a type of full analysis, the results can be considered valid and useful. The four indices of the results were: 1. total force due to the friction stress (CFSM); 2. frictional dissipation (FD): the frictional energy dissipation of the whole model; 3. strain energy (SE): the strain energy of the whole model; 4. maximum von Mises stress (Max): the maximum von Mises stress of the whole model.

For the above results, $F$-test (analysis of variance) was used to determine the significant difference under different modeling conditions. The level of statistical significance was set to $P<0.05$.

\section{Results}

\subsection{Frictional behavior when endoscope passed through the narrow region}

Given the structure and the interfacial property affecting the frictional behavior at the endoscopeesophagus interface, the radius and the length of the narrowest position, the coefficient of friction and the amplitude were studied in this section.

\subsubsection{Effect of radius}

As shown in Fig. 5, there is a clear difference concerning the strain energy of the whole model $(P<0.05)$. With the increasing radius of the narrowest position, the interfacial tightness declines following a decreasing energy when the endoscope passes through the narrow region, namely strain energy. Meanwhile, the total force duo to the frictional force and the corresponding frictional dissipation of the whole model have no significant difference. That is mainly due to the short contact length of the narrowest position relative to the overall system. According to Figs. $5 \mathrm{~d}$ and 6 , the radius at the narrowest position is negatively correlated with the maximum von Mises stress, with an increase of nearly $25 \%$ from $3.75 \mathrm{~mm}$ to $3.25 \mathrm{~mm}$ in radius.

\subsubsection{Effect of length}

For the different lengths of the narrowest position, it is interesting to note that the results concerning the total force duo to the frictional force, frictional dissipation, strain energy and the maximum von Mises stress for the whole model (Figs. 7a-d and 8) are almost identical when compared with the results in section 3.1.1 $(P>0.05)$. On the one hand, the length 
Fig. 5. The results of model under different radii of the narrowest position: (a) total force duo to the friction force (CFSM), (b) frictional dissipation (FD), (c) strain energy (SE), (d) maximum von Mises stress (Max)

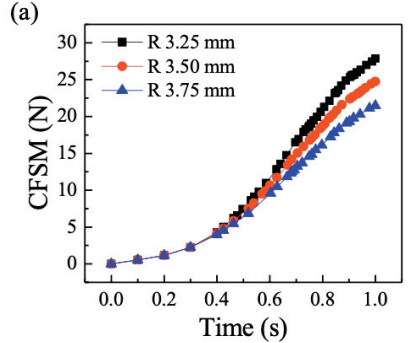

(c)

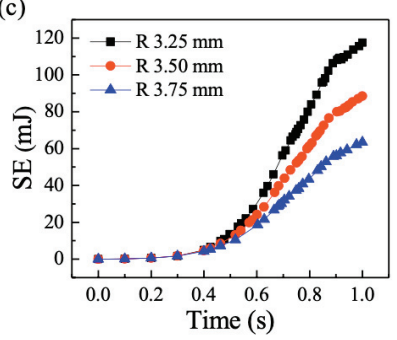

(b)

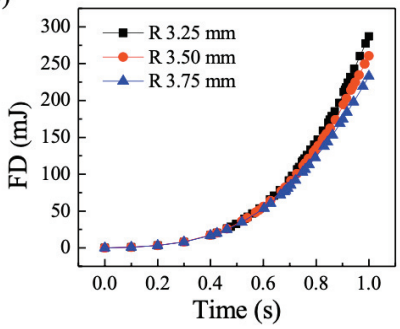

(d)

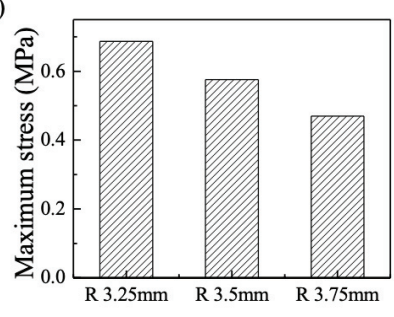

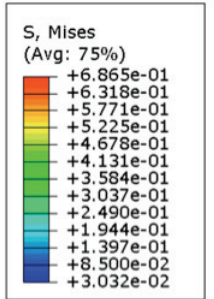

$\underset{\mathbf{x}}{\longleftarrow}$
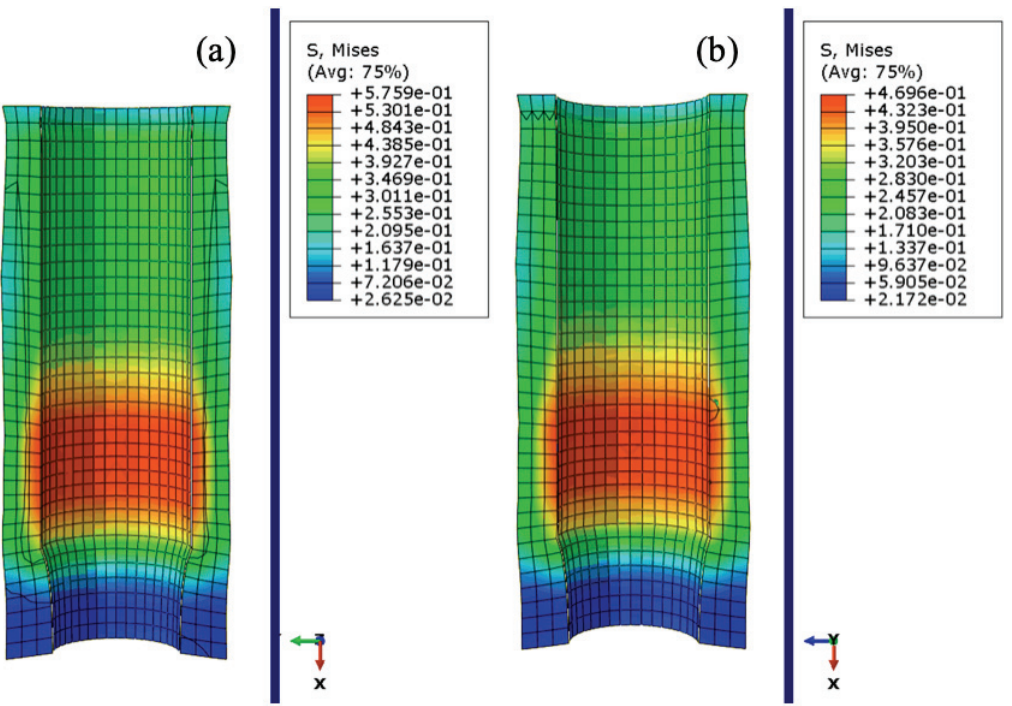

(c)

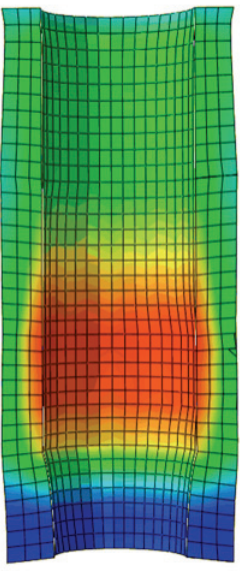

Fig. 6. The stress contour of narrow region under different radii of the narrowest position:

(a) $R=3.25 \mathrm{~mm}$, (b) $R=3.5 \mathrm{~mm}$; (c) $R=3.75 \mathrm{~mm}$

Fig. 7. The results of model under different lengths of the narrowest position: (a) total force duo to the friction force (CFSM); (b) frictional dissipation (FD); (c) strain energy (SE); (d) maximum von Mises stress (Max)

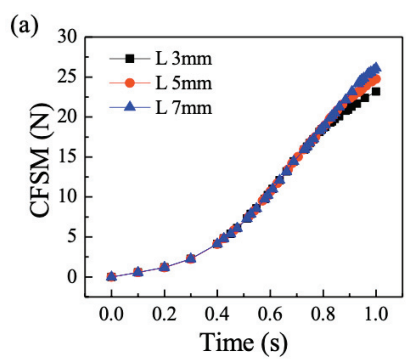

(c)

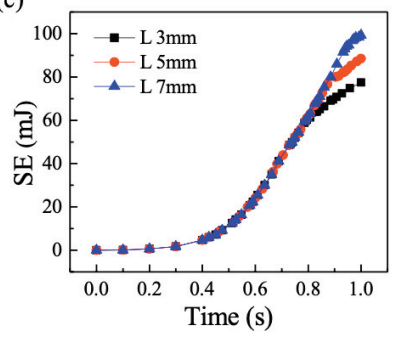

(b)

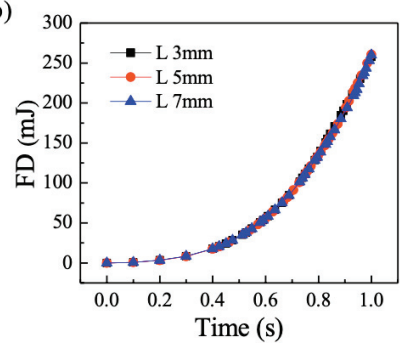

(d)

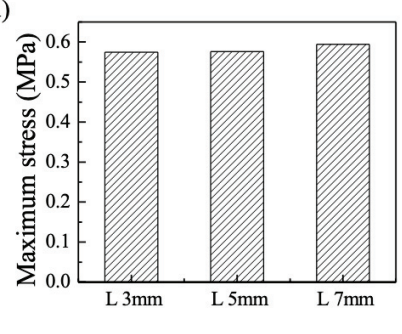




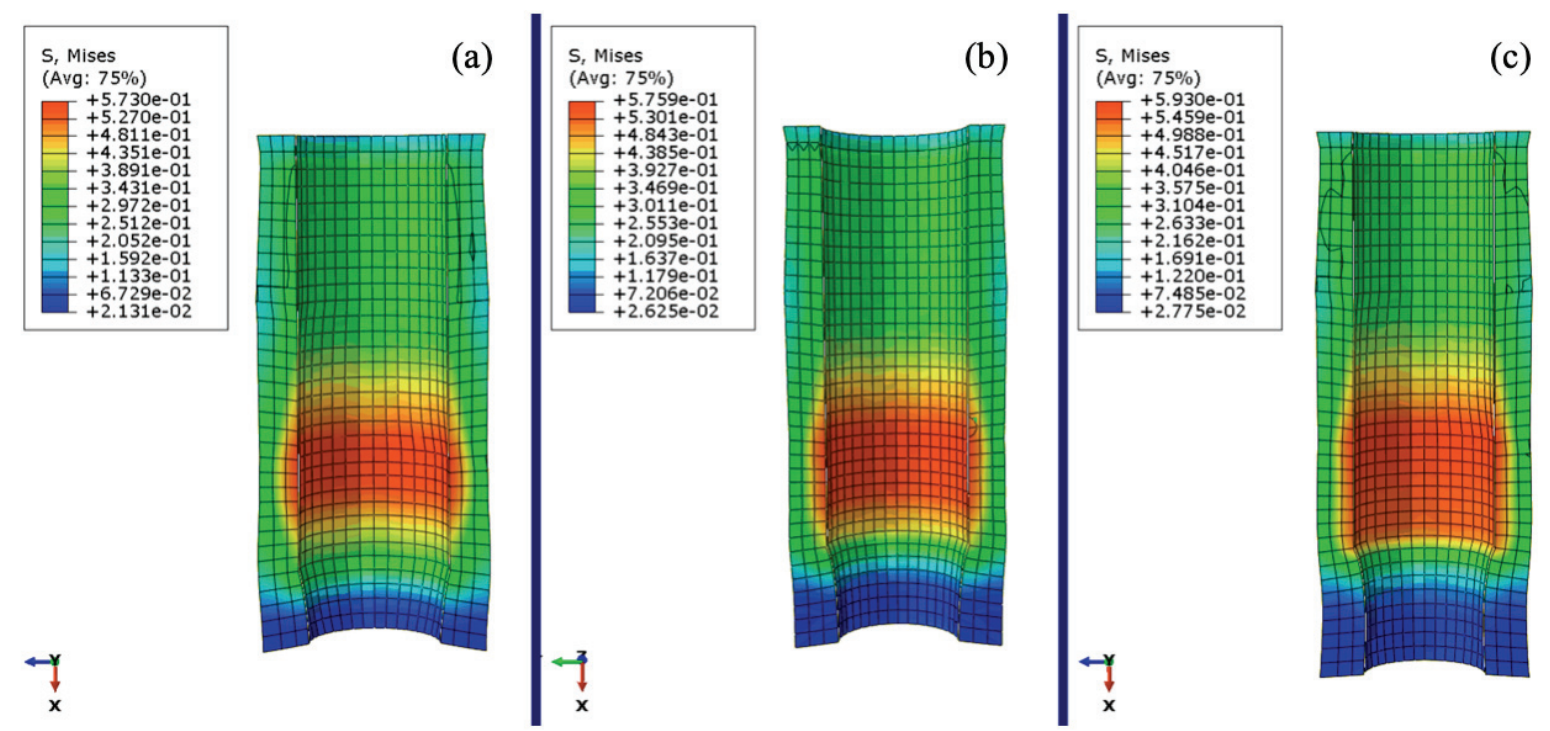

Fig. 8. The stress contour of narrow region under different lengths of the narrowest position: (a) $L=3 \mathrm{~mm}$, (b) $L=5 \mathrm{~mm}$, (c) $L=7 \mathrm{~mm}$

of the narrowest position is less than the total length of the esophagus in the narrow region, as shown in Fig. 19 in Appendix; on the other hand, the results illustrate that the radius at the narrowest position has a great impact on the strain energy and the maximum von Mises stress of the whole model, especially on the latter, which is related to the damage of biological tissue during endoscopy [18].

\subsubsection{Effect of COF}

As can be seen in Figs. 9a and b, along with the increasing $\mathrm{COF}$ at the endoscope-esophagus interface, there is a clear growing tendency for the total force duo to the frictional force and the frictional dissipation $(P<0.05)$. In the initial stage before contacting the narrowest position, the strain energy of the whole model approximately coincides. When the endoscope passes through the narrowest position, the system needs more strain energy to overcome the resistance caused by the larger COF. Besides, although the maximum von Mises stress increases slightly, the entire stress distribution exists a difference when the COF is 0.3 , as shown in Fig. 11c. Except for the usual stress concentration at the narrowest position, the higher COD increases the stress at the proximal esophageal interface. (a)

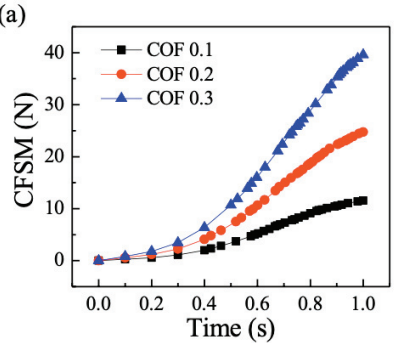

(c)

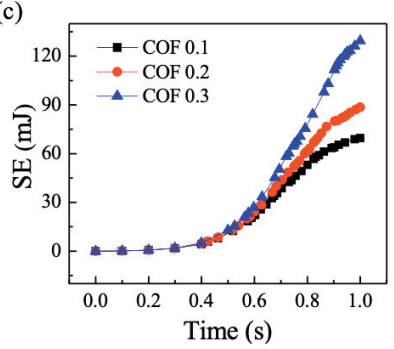

(b)

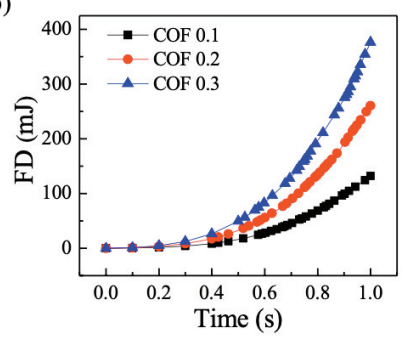

(d)

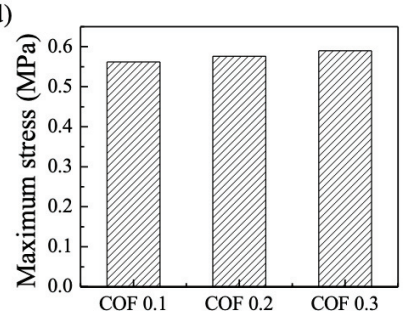

Fig. 9. The results of model under different coefficient of friction: (a) total force duo to the friction force (CFSM),

(b) frictional dissipation (FD), (c) strain energy (SE), (d) maximum von Mises stress (Max) 


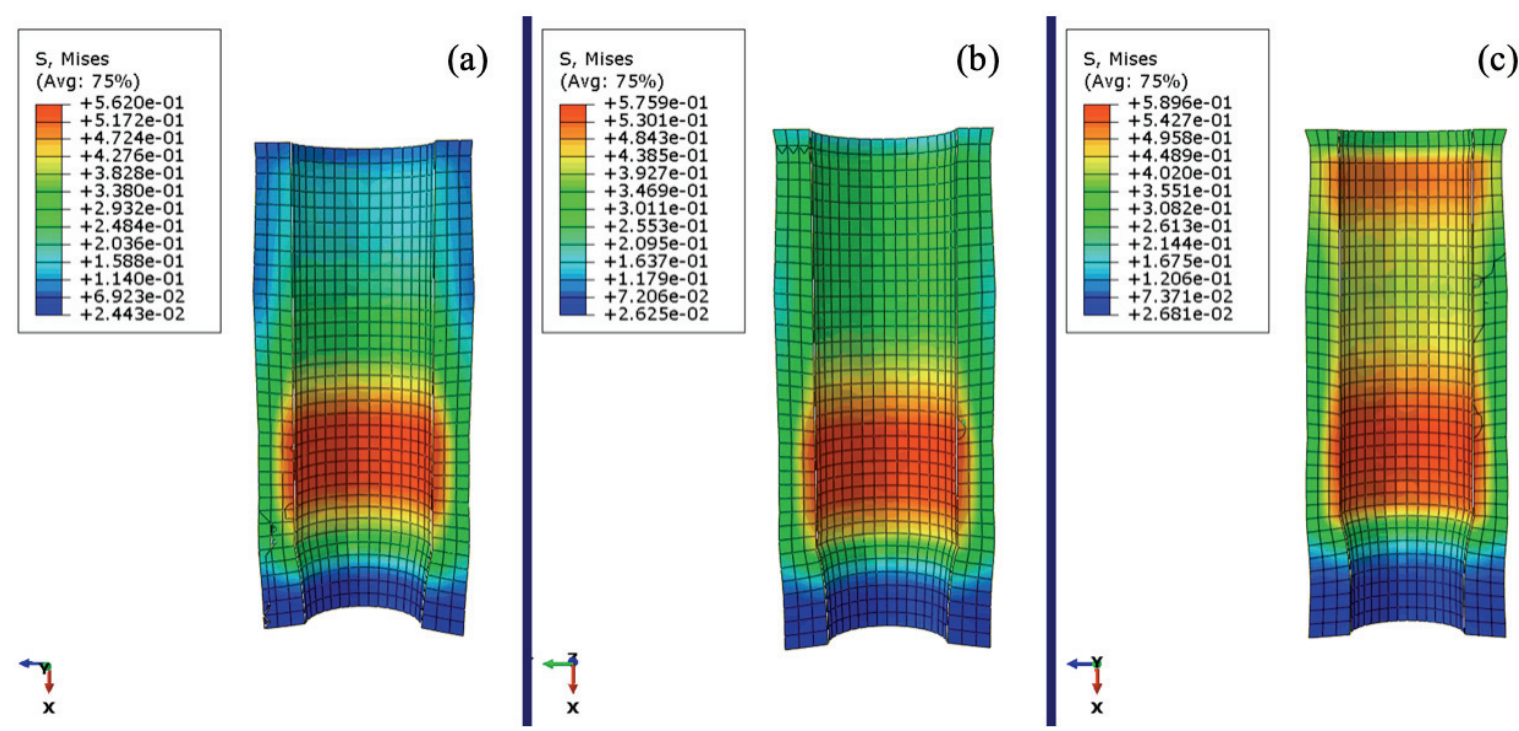

Fig. 10. The stress contour of narrow region part under different $\mathrm{COF}$ of narrow region:

(a) $\mathrm{COF}=0.1$, (b) $\mathrm{COF}=0.2$, (c) $\mathrm{COF}=0.3$

\subsubsection{Effect of amplitude}

In this section, the amplitudes are respectively: amplitude 1: $(0,0)-(1,1)$, amplitude 2: $(0,0)-$ $(1,1.5)$ and amplitude $3:(0,0)-(1,2)$. The amplitude in the model is used to control the displacement of the endoscope. For example, the displacement of endoscope was $30 \mathrm{~mm}$ the amplitude $(0,0)-(1,1)$ means that the displacement of endoscope is $0 \mathrm{~mm}$ at $0 \mathrm{~s}$ and $30 \mathrm{~mm}$ at $1 \mathrm{~s}$. Similarly to the dependence of frictional behavior of biological tissue on velocity, the total force duo to the frictional force, frictional dissipation and the strain energy of the whole model all increase with the growing amplitude of the model, as shown in Figs. 11a-c $(P<0.05)$. In addition, the CFSM and SE present a straight line segment at the end of the curve due to the fact that the endoscope has reached the end of the esophagus, as shown in Figs. 11a and c. However, as the frictional dissipation is an accumulation process, there is no "straight line" phenomenon for the curves in Fig. 11b. Meanwhile, there is not a significant difference in the maximum von Mises stress according to the results in Figs. 11d and 12.
Fig. 11. The results of model under different amplitudes: (a) total force duo to the friction force (CFSM),

(b) frictional dissipation (FD); (c) strain energy (SE); (d) maximum von Mises stress (Max) (a)

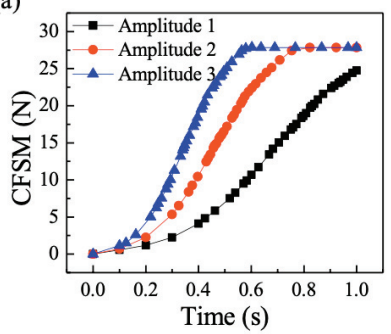

(c)

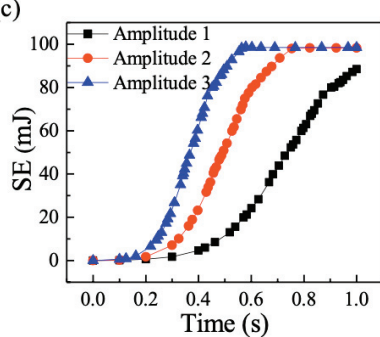

(b)

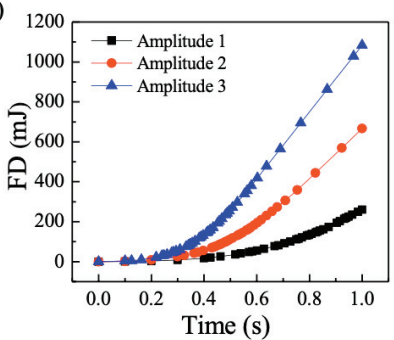

(d)

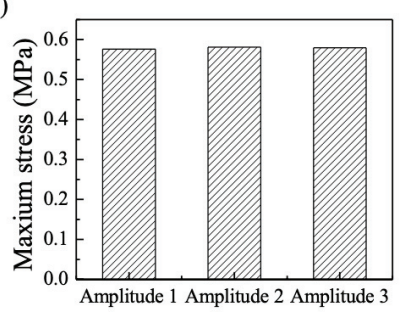




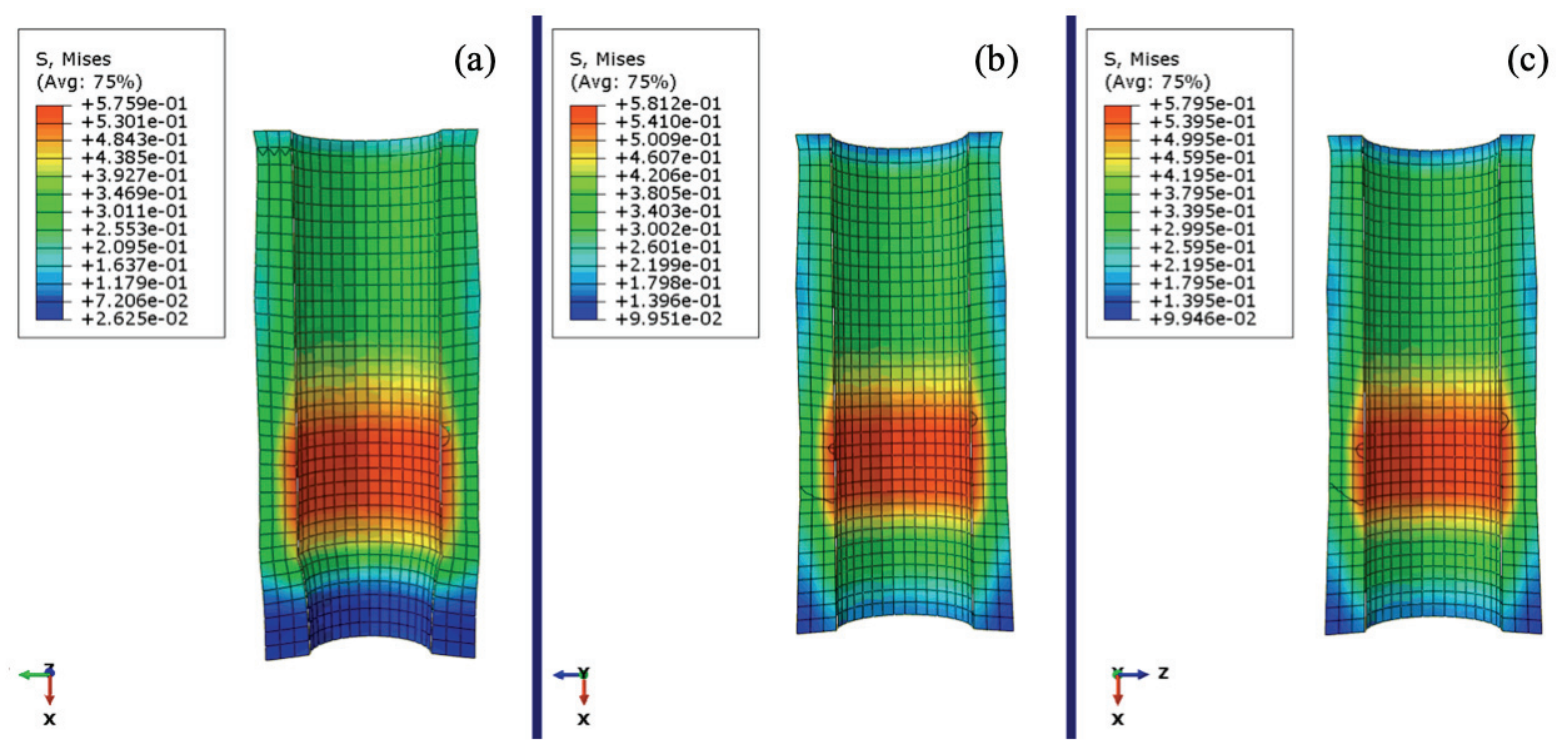

Fig. 12. The stress contour of narrow region under different amplitudes:

(a) amplitude 1, (b) amplitude 2, (c) amplitude 3

\subsection{Frictional behavior when endoscope passed through the abdominal region}

\subsubsection{Effect of COF}

As shown in Figs. 13a-d, along with the increasing $\mathrm{COF}$ at the endoscope-abdominal region interface, the total force duo to the frictional force, frictional dissipation, strain energy and the maximum von Mises stress present an obvious rising trend $(P<0.05)$. It's a process of expansion when the endoscope enters the abdominal esophagus according to the size of the endoscope and the esophagus in Appendix. At the same time, the maximum von Mises stress increases from 16 to $40 \%$ along with the increase of COF from 0.1 to 0.3 . The region of stress concentration also presents a difference. The stress region gradually accumulates in the initial stage, as shown in Fig. 14.
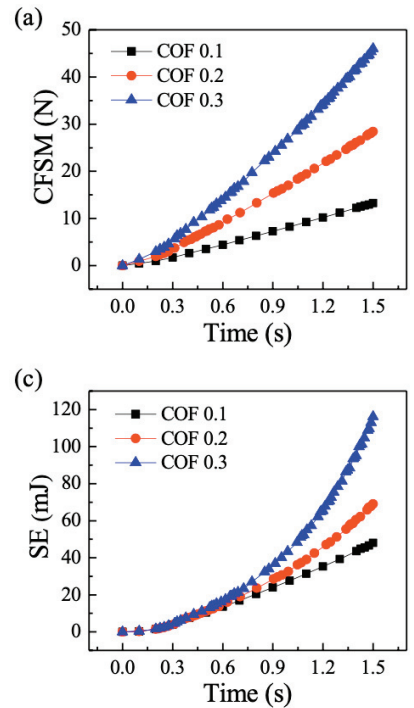

(b)

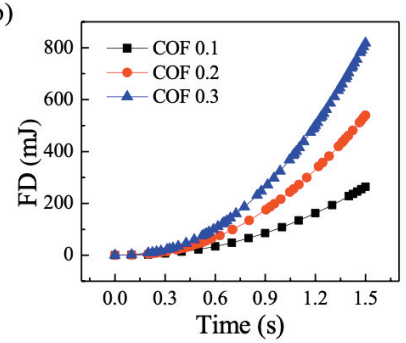

(d)

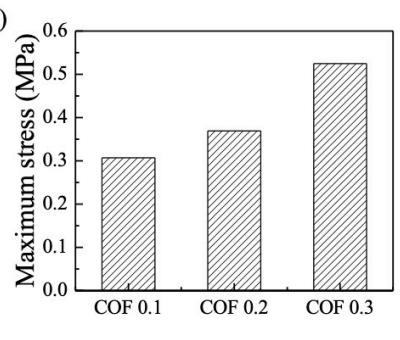

Fig. 13. The results model under different COF: (a) total force duo to the friction force (CFSM), (b) frictional dissipation (FD), (c) strain energy (SE), (d) maximum von Mises stress (Max) 

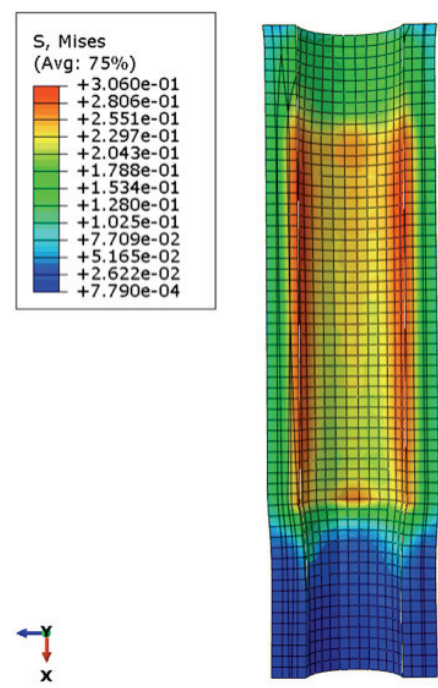

(a)

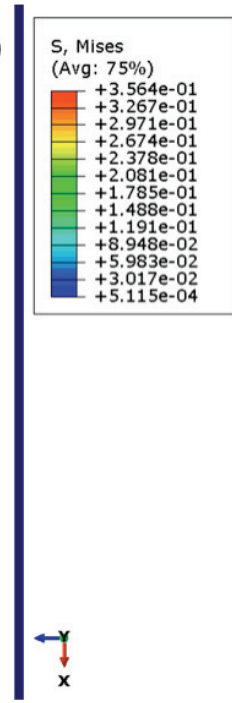

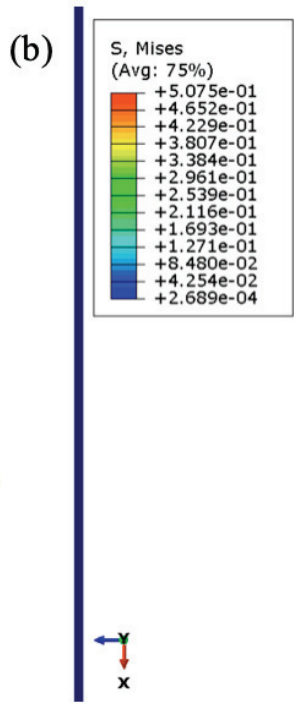

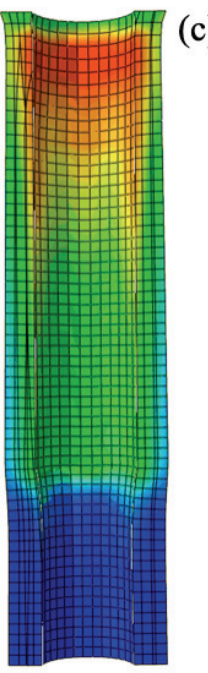

(c)

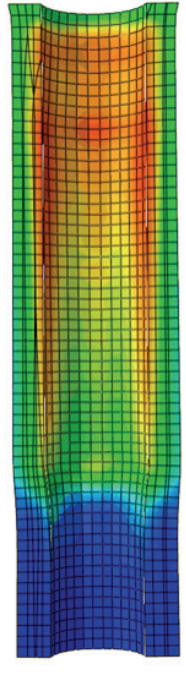

Fig. 14. The stress contour of abdominal region under different COF:

(a) $\mathrm{COF}=0.1$, (b) $\mathrm{COF}=0.2$, (c) $\mathrm{COF}=0.3$

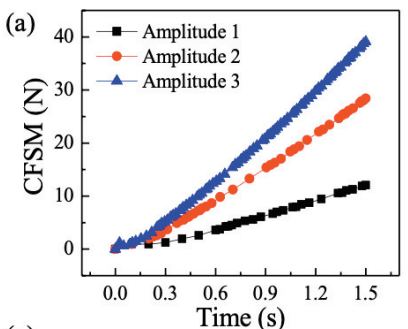

(c)

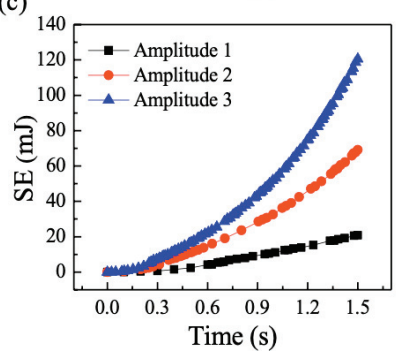

(b)

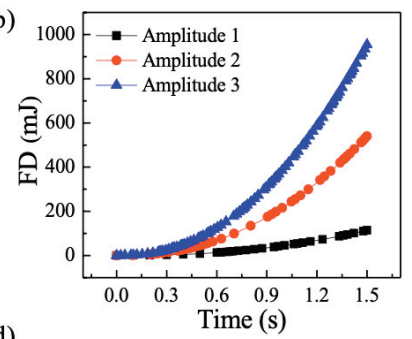

(d)

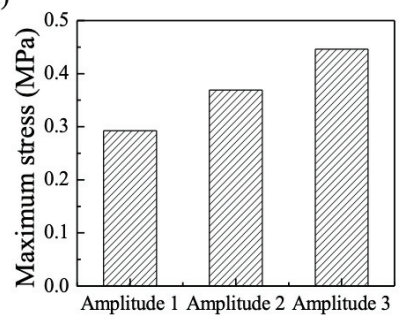

Fig. 15. The results of model under different amplitudes: (a) total force duo to the friction force (CFSM), (b) frictional dissipation (FD), (c) strain energy (SE), (d) maximum von Mises stress (Max)
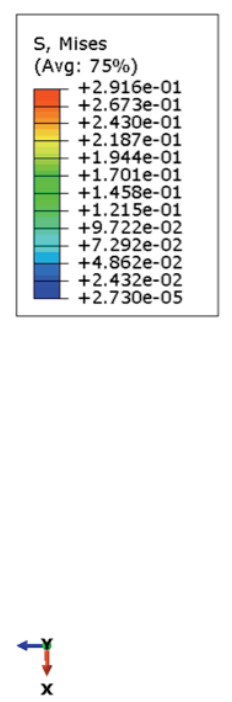

(a)

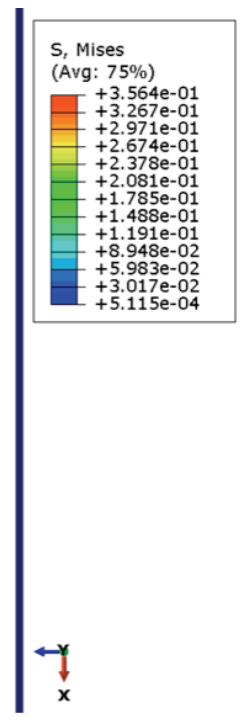

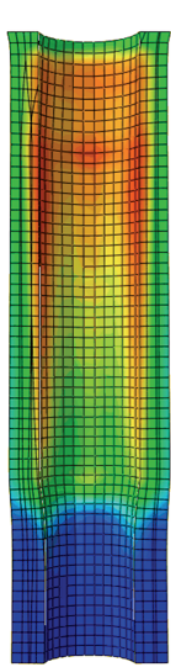

(b)

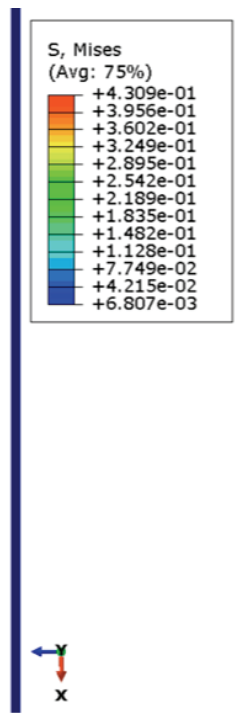

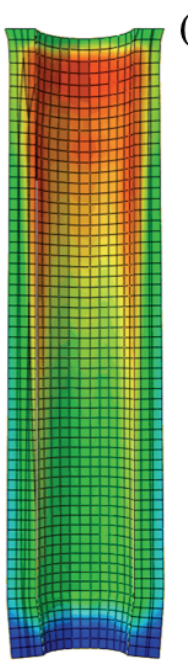

(c)

Fig. 16. The stress contour of abdominal region under different amplitudes:

(a) amplitude 1, (b) amplitude 2, (c) amplitude 3 


\subsubsection{Effect of amplitude}

The amplitudes in this section are also variable: amplitude 1: $(0,0)-(1.5,0.5)$, amplitude $2:(0,0)-(1.5,1)$ and amplitude 3: $(0,0)-(1.5,1.3)$. As shown in Figs. 15a-c, the total force duo to the frictional force, frictional dissipation, strain energy and the maximum von Mises stress are significantly related to the amplitude $(P<0.05)$. Compared to the results in Fig. 13a-c, all curve shapes of abdominal esophagus are similar. When the amplitude is smaller, the region of stress concentration is mainly focuses on the distal end of contact region; in contrast, the stress region gradually accumulates in the initial stage with the larger amplitude, as shown in Fig. 16. The value of maximum von Mises stress is also different from Fig. 15d.

\subsection{Frictional behavior when endoscope passed through the thoracic region}

Because the trachea is adjacent to the aorta, the esophagus presents an inflection point at the joint region. As a result, there is an additional impact point on the esophageal wall compared to the previous esophageal regions. On the one hand, along with the increasing COF, the total force duo to the frictional force, frictional dissipation, strain energy and the maximum von Mises stress all augment in Fig. 17 $(P<0.05)$. Meanwhile, the position of maximum von Mises stress is gradually approaching the initial stage, according to the Fig. 18. On the other hand, for the
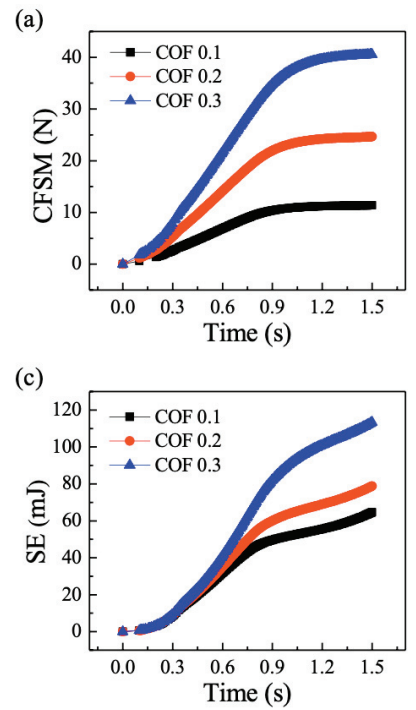

(b)

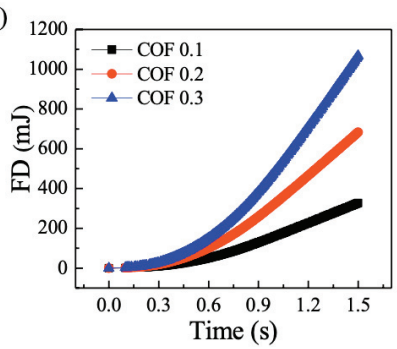

(d)

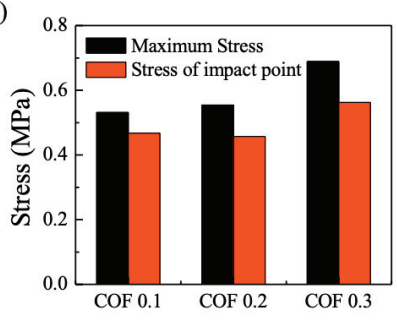

Fig. 17. The results of model under different COF:

(a) total force duo to the friction force (CFSM),

(b) frictional dissipation (FD), (c) strain energy (SE),

(d) maximum von Mises stress (Max)

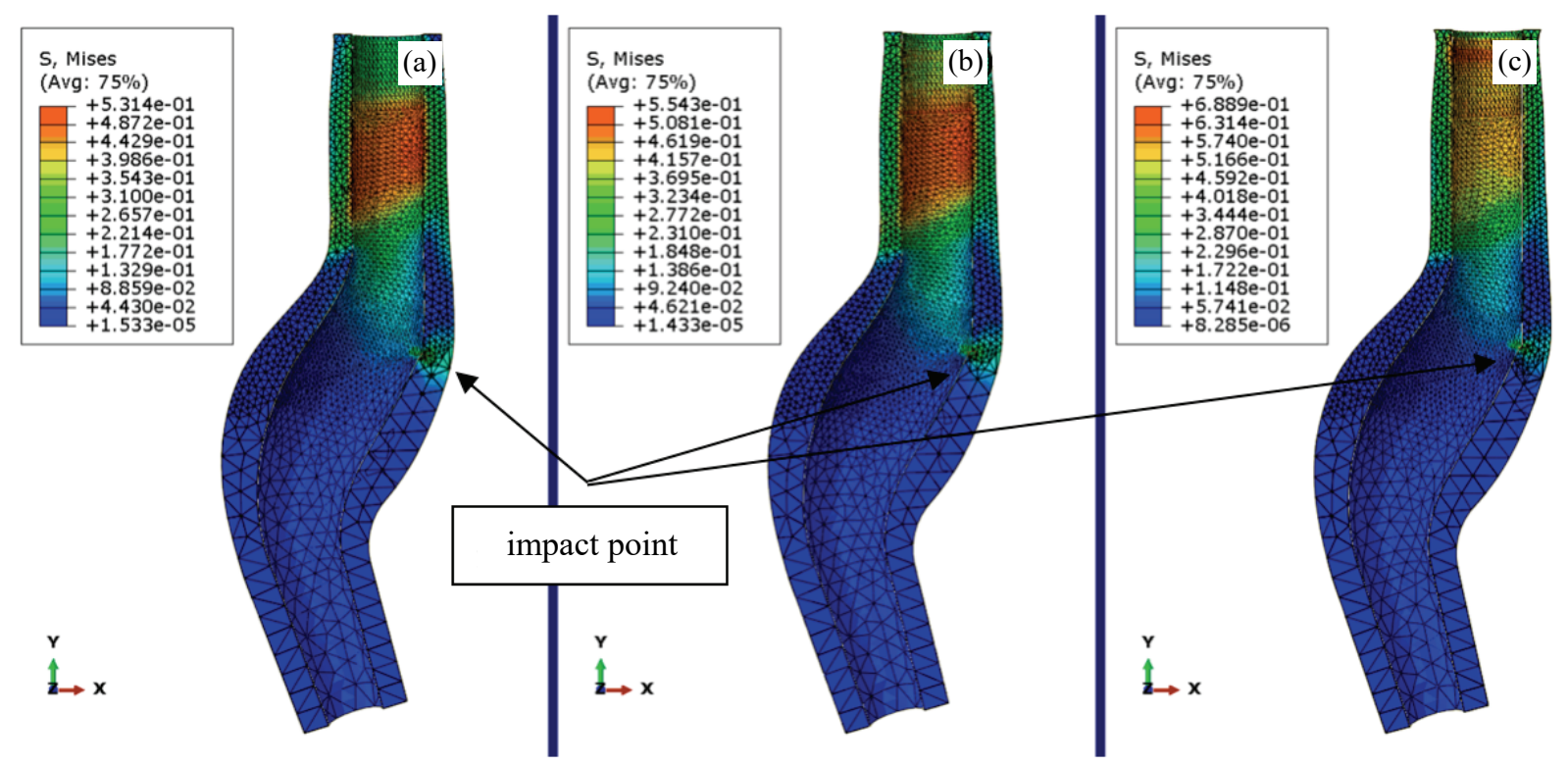

Fig. 18. The stress contour of thoracic region under different COF:

(a) $\mathrm{COF}=0.1$, (b) $\mathrm{COF}=0.2$, (c) $\mathrm{COF}=0.3$ 
stress value of the impact point on the esophageal wall, the difference between the former two COF is not significant. When the COF reaches 0.3 , the stress at the impact point and the maximum von Mises stress both appear significant variation. The ratio of the stress at the impact point to the maximum stress is close to $81-86 \%$.

\section{Discussion}

As a usually overlooked problem, the relationship between medical operation and contact force and stress distribution during endoscopy is unclear. In current paper, the frictional behaviors between the endoscope and the esophagus were studied by finite element model considering a patient-specific model.

As we know, there are three natural narrow regions for a healthy human esophagus, which are variable in size, namely radius and length. For narrowregion models with different radii, lengths, COF and amplitudes, the frictional behaviors were different. As shown in Fig. 5, the maximum von Mises stress presented a significant difference with the decreasing radius, which was a significant indicator of tissue damage during endoscopy [15]. That was also an interesting phenomenon found in other experiments concerning capsule endoscope [14], [28], [29]. According to Kim's theory [13], the hoop stress increased with the increasing inner pressure. It was similar to the theory that a decrease in esophageal diameter leaded to an increase in inner pressure with the constant endoscope diameter. Relative to the other factors such as length, COF and amplitude (Figs. 7, 9, 11 and 13), radius was the dominant factor in stress concentration. As a result, it is of great significance to choose the appropriate type of the endoscope for specific patient before endoscopy.

Meanwhile, the results of CFSM, FD and SE were not significantly different for different narrow-region structure parameters (Figs. 7, 8). This was mainly because the length of the narrowest region, as shown in Fig. 19 (Appendix), was relatively small in proportion to the length of the whole esophagus. When the fronttip of the endoscope passed through the narrowest position of the esophagus, it almost reached the preset position. So it didn't have much effect on the whole frictional behavior. Besides, it can be seen that the CFSM and FD both increased directly with the increasing COF (Figs. 9a and b). In order to overcome the large resistance duo to frictional force, the model need more strain energy to move forward (Fig. 9c).
Meanwhile, CFSM, FD and SE also had amplitude dependence (Figs. 11a-c), which was also a typical phenomenon for other soft tissues, such as esophagus [15], [16], intestine [28] and skin [27].

In contrast, along with the increasing $\mathrm{COF}$ and amplitude, CFSM, FD and SE in abdominal region and thoracic region had the similar trends, as shown in Figs. 13a-c, 15a-c and $17 \mathrm{a}-\mathrm{c}$. Depending on the size of the abdominal region in Fig. 20 (Appendix) and thoracic region in Fig. 21 (Appendix), the endoscope both went through a process of sustained compression starting from the initial buffer zones. Therefore, CFSM, FD and SE changed obviously owning to the incremental contact area. In addition, it can also be observed in Figs. 14a, 16a and 18a that the interfacial stress was distributed in the extrusion area. When the loading environment worsen, that is, the COF or amplitude increased, there existed a difference for value of the von Mises stress as well as the distribution region, especially the former. On the one hand, the thickness of the buffer zone (Figs. 20 and 21) was less than the normal thickness of the esophagus. On the other hand, once the endoscope started to pass the esophagus, the esophagus near the buffer region was in a persistent and cumulative contact. So the stress concentration region gradually approached the initial contact stage and had a larger COF or amplitude.

It is an interesting structure for the thoracic esophagus which was different from the routine anatomic diagram of human esophagus [6]. There was an impact point on the esophageal wall. The proportion of the impact stress and the maximum stress approached $81-86 \%$. Compared to the above behavior of direct contact between the endoscope and the esophagus wall above, it is a easily neglected problem. So it is better for medical workers to slow down the speed of the endoscope and improve the lubrication environment when the endoscope passes through the thoracic esophagus during endoscopy.

The above results can provide theoretical basis and technical support for clinical application. It can also offer some suggestions for medical workers during endoscopy. There are, of course, still some improvements to the current model. Firstly, the model does not take the liquid environment into account. The human internal environment maintains a relatively stable state with plenty of water. Meanwhile, the constitutive equation for esophagus can be modified more precisely to improve the similarity and accuracy. Thirdly, in vivo friction experiment is the research direction of our research group in the next stage. 


\section{Conclusions}

Considering the physiological structure of esophagus in a specific patient, this paper selected three characteristic regions to study the frictional behavior between the endoscope and the esophagus and drew the following conclusions:

1. The radius of the narrowest position was the dominant factor of the maximum von Mises stress when the endoscope passed through the narrow region.

2. With the increasing COF and amplitude, the CFSM, FD, SE and maximum von Mises stress in abdominal region and thoracic region all increased accordingly; meanwhile, the stress concentration region gradually approached the initial contact stage.

3. With the increasing COF, the stress value of the impact point to the wall of the thoracic esophagus also augmented.

\section{Acknowledgement}

This work was supported by National Natural Science Foundation of China (Nos. 51675447).

\section{Reference}

[1] Andreoni B., Farina M.L., Biffi R., Crosta C., Esophageal perforation and caustic injury: emergency management of caustic ingestion, Dis. Esophagus, 1997, 10 (2), 95-100, DOI: 10.1093/dote/10.2.95.

[2] Athanassiadi K., Gerazounis M., Metaxas E., Kalantzi N., Management of esophageal foreign bodies: a retrospective review of 400 cases, Eur. J. Cardiothorac. Surg., 2002, 21 (4), 653-656, DOI: 10.1016/S1010-7940(02)00032-5.

[3] Auricchio F., Conti M., Ferrara A., Morganti S., Reali A., Patient-specific finite element analysis of carotid artery stenting: a focus on vessel modeling, Int. J. Numer Method Biomed. Eng., 2013, 29 (6), 645-664, DOI: 10.1002/cnm.2511.

[4] Auricchio F., Conti M., Morganti S., Reali A., Simulation of transcatheter aortic valve implantation: a patientspecific finite element approach, Comput. Methods Biomech. Biomed. Engin., 2014, 17 (12), 1347-1357, DOI: 10.1080/ 10255842.2012.746676.

[5] Auricchio F., Di Loreto M., SACCo E.. Finite-element Analysis of a Stenotic Artery Revascularization Through a Stent Insertion, Computer Methods in Biomechanics and Biomedical Engineering, 2001, 4 (3), 249-263, DOI: 10.1080/10255840108908007.

[6] Bouma B.E., Tearney G.J., Compton C.C., Nishioka N.S., Highresolution imaging of the human esophagus and stomach in vivo using optical coherence tomography, Gastrointestinal Endoscopy, 2000, 51 (4), 467-474, DOI: 10.1016/S0016-5107(00)70449-4.

[7] Carniel E.L., Frigo A., Fontanella C.G. et al., A biomechanical approach to the analysis of methods and procedures of bariatric surgery, J. Biomech., 2017, 56, 32-41, DOI: 10.1016/j.jbiomech.2017.02.029.
[8] Dai Y., Chopra S.S, Steinbach M., Knief S., Hunerbein M., Esophageal stents for leaks and perforations, Semin. Thorac. Cardiovasc. Surg., 2011, 23 (2), 159-162, DOI:10.1053/ j.semtcvs.2011.08.004.

[9] DotTORi S., Flamini V., VAIRo G., Mechanical behavior of peripheral stents and stent-vessel interaction: A computational study, International Journal for Computational Methods in Engineering Science \& Mechanics, 2016, 17 (3), 196-210, DOI: $10.1080 / 15502287.2016 .1188530$.

[10] Eroglu A., Turkyilmaz A., Subasi M., Karaoglanoglu N., The use of self-expandable metallic stents for palliative treatment of inoperable esophageal cancer, Dis. Esophagus, 2010, 23 (1), 64-70, DOI: 10.1111/j.1442-2050.2009.00978.x.

[11] Giovannini M., Monges G., Seitz J.F. et al., Distant lymph node metastases in esophageal cancer: impact of endoscopic ultrasound-guided biopsy, Endoscopy, 1999, 31 (7), 536-540, DOI: 10.1055/s-1999-60.

[12] Kajzer W., Kaczmarek M., Marciniak J., Biomechanical analysis of stent-oesophagus system, Journal of Materials Processing Technology, 2005, 162-163, 196-202, DOI: 10.1016/ j.jmatprotec.2005.02.209.

[13] KIm J.S., Sung I.H., KIM Y.T., Kim D.E., JANG Y.H., Analytical model development for the prediction of the frictional resistance of a capsule endoscope inside an intestine, Proc. Inst. Mech. Eng. H., 2007, 221 (8), 837-845, DOI: 10.1243/09544119JEIM173.

[14] KwOn J., PARK S., Kim B., PARK J.O., Biomaterial property measurement system for locomotive mechanism in gastrointestinal tract, Proceedings of the 2005 IEEE International Conference on Robotics and Automation, 2005, 1303-1308.

[15] Lin C.X., Li W., Deng H.Y., Li K., Zhou Z.R., Friction Behavior of Esophageal Mucosa Under Axial and Circumferential Extension, Tribology Letters, 2019, 67 (1), 9, DOI: 10.1007/s11249-018-1123-x.

[16] Lin C.X., Yu Q.Y., Wang J., Ji W., Li W., Zhou Z.R., Friction behavior between endoscopy and esophageal internal surface, Wear, 2017, 376, 272-280, DOI: 10.1016/ j.wear.2016.11.011.

[17] Little D.C., Shah S.R., St Peter S.D., Calkins C.M. et al., Esophageal foreign bodies in the pediatric population: our first 500 cases, J. Pediatr. Surg., 2006, 41 (5), 914-918, DOI: 10.1016/j.jpedsurg.2006.01.022.

[18] Li W., SHI L., DeNG H.Y., Zhou Z.R., Investigation on Friction Trauma of Small Intestine In Vivo Under Reciprocal Sliding Conditions, Tribology Letters, 2014, 55 (2), 261-270, DOI: 10.1007/s11249-014-0356-6.

[19] MaGne P., Efficient 3D finite element analysis of dental restorative procedures using micro-CT data, Dent. Mater., 2007, 23 (5), 539-548, DOI: 10.1016/j.dental.2006.03.013.

[20] Migliavacca F., Petrini L., Massarotti P., Schievano S., AuricChIo F., Dubini G., Stainless and shape memory alloy coronary stents: a computational study on the interaction with the vascular wall, Biomech. Model Mechanobiol., 2004, 2 (4), 205-217, DOI: 10.1007/s10237-004-0039-6.

[21] Morganti S., Auricchio F., Benson D.J. et al., Patient-specific isogeometric structural analysis of aortic valve closure, Computer Methods in Applied Mechanics and Engineering, 2015, 284, 508-520, DOI: 10.1016/j.cma.2014.10.010.

[22] Mozafari H., Dong P., Zhao S., Bi Y., Han X., Gu L., Migration resistance of esophageal stents: The role of stent design, Comput. Biol. Med., 2018, 100, 43-49, DOI: 10.1016/ j.compbiomed.2018.06.031.

[23] Ni X.Y., Pan C.W., Gangadhara Prusty B., Numerical investigations of the mechanical properties of a braided non- 
-vascular stent design using finite element method, Comput. Methods Biomech. Biomed. Engin., 2015, 18(10), 1117-1125, DOI: 10.1080/10255842.2013.873420.

[24] Ni X.Y., Zhang Y.H., ZHAO H.X., PAN C.W., Numerical research on the biomechanical behaviour of braided stents with different end shapes and stent-oesophagus interaction, Int. J. Numer Method. Biomed. Eng., 2018, 34 (6), e2971, DOI: $10.1002 / \mathrm{cnm} .2971$.

[25] Schiavone A., Abunassar C., Hossainy S., Zhao L.G., Computational analysis of mechanical stress-strain interaction of a bioresorbable scaffold with blood vessel, J. Biomech., 2016, 49 (13), 2677-2683, DOI: 10.1016/j.jbiomech.2016.05.035.

[26] Schiavone A., Zhao L.G., ABDel-WahaB A.A., Effects of material, coating, design and plaque composition on stent deployment inside a stenotic artery--finite element simulation, Mater Sci. Eng. C. Mater. Biol. Appl., 2014, 42, 479-88, DOI: 10.1016/j.msec.2014.05.057.

[27] TANG W., Gel S.R., Zhu H., CaO X.C., Li N., The influence of normal load and sliding speed on frictional properties of skin, Journal of Bionic Engineering, 2008, 5 (1), 33-38, DOI: 10.1016/S1672-6529(08)60004-9.

[28] Wang X., Meng M.Q., An experimental study of resistant properties of the small intestine for an active capsule endoscope, Proc. Inst. Mech. Eng. H., 2010, 224 (1), 107-18, DOI: 10.1243/09544119JEIM540.

[29] Zhang C., Liu H., Li H.Y., Experimental investigation of intestinal frictional resistance in the starting process of the capsule robot, Tribology International, 2014, 70, 11-17, DOI: 10.1016/j.triboint.2013.09.019.

[30] Zhao H., LiU Y., Ni X., Xia F., Zhang X., Mechanical Performance of Cup-spherical-shaped and Straight Form Braided Esophageal Stent, TELKOMNIKA Indonesian Journal of Electrical Engineering, 2013, 11(11), 6657-6663, DOI: 10.11591/telkomnika.v11i11.3512.

\section{Appendix}

The basic parameters of different esophageal region are below, which is drown in AutoCAD 2016 (Autodesk, USA):

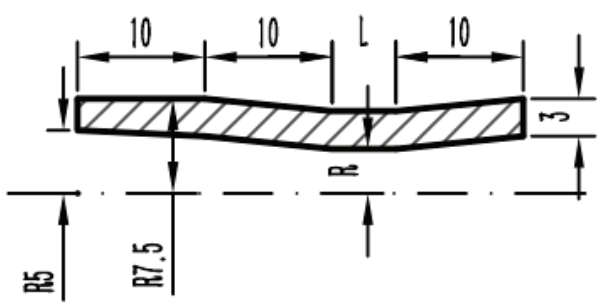

Fig. 19. The size of the narrow region in the model of Abaqus
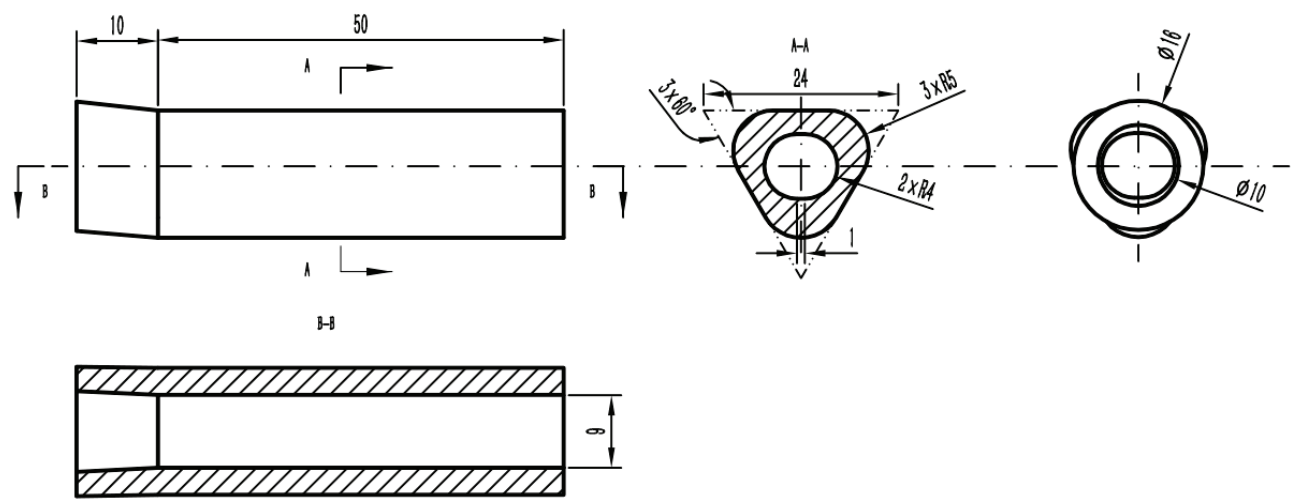

Fig. 20. The size of the abdominal region in the model of Abaqus

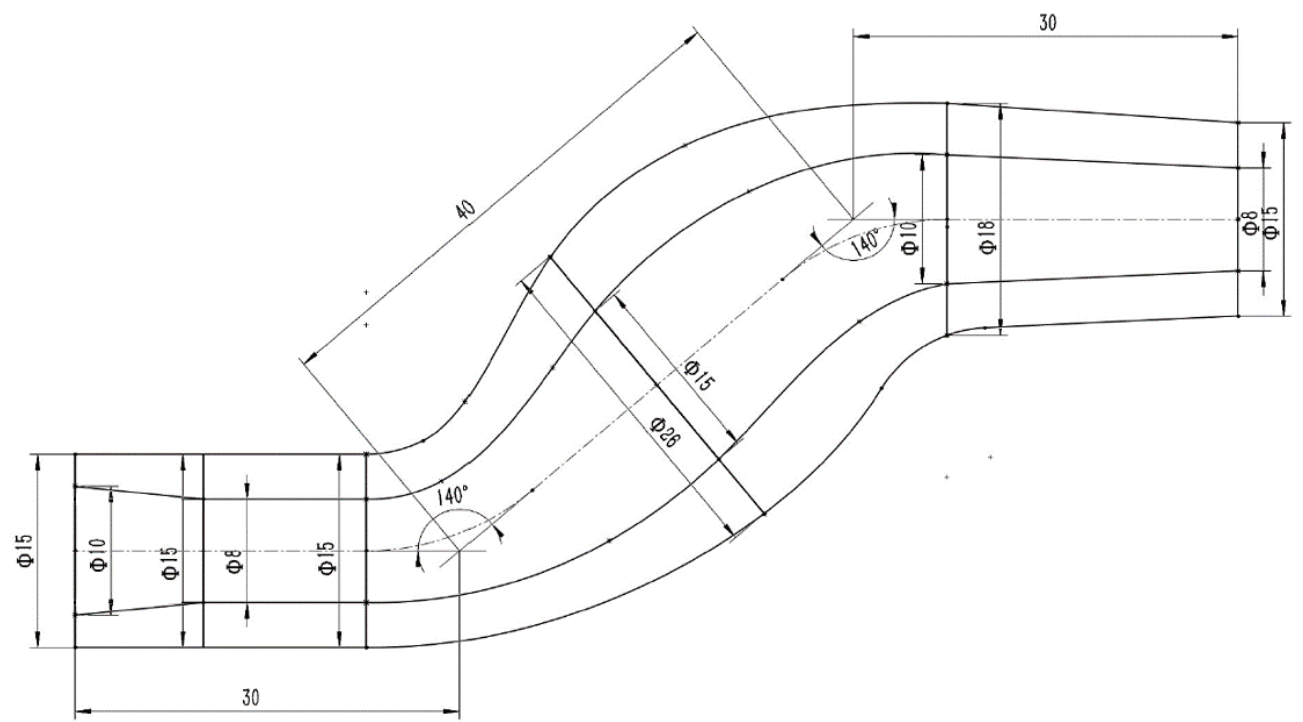

Fig. 21. The size of the thoracic region in the model of Abaqus 


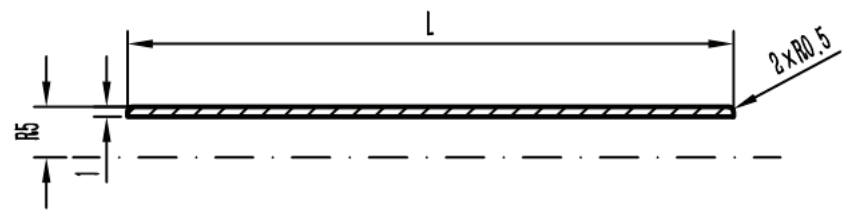

Fig. 22. The size of the endoscope in the model of Abaqus ( $L$ is $100 \mathrm{~mm}$ for thoracic region and $60 \mathrm{~mm}$ for other two regions) 